\title{
Reincorporación laboral de mujeres con cáncer de mama: experiencia de un centro oncológico en la ciudad de Medellín
}

Return to work after breast cancer: experience of an oncological referral centre in Medellín, Colombia

\author{
Elsa Maria Vasquez-Trespalacios ${ }^{1}$ \\ Sara Atehortua-Salazar ${ }^{1}$ \\ Daniela Arango-Isaza' \\ Clara Paulina Gallego Vélez ${ }^{1}$ \\ Luis Javier Gallón Villegas ${ }^{1}$ \\ ${ }^{1}$ Facultad de Medicina, Universidad CES, Medellín, Colombia.
}

Fechas - Dates

Recibido: 2019.11.25

Aceptado: 2020.04.14

Publicado: 2020.07.15
Correspondencia · Corresponding Author

Elsa Maria Vasquez Trespalacios

e-mail: evasquez@ces.edu.co 


\title{
Resumen
}

Introducción: los avances en el diagnóstico y tratamiento del cáncer de mama han mejorado el pronóstico para estas pacientes, por lo tanto, se espera que un mayor número de supervivientes se enfrenten con el proceso de retornar al trabajo. El objetivo del presente estudio es analizar la frecuencia, la mediana del tiempo, así como los factores relacionados con el retorno al trabajo de pacientes con cáncer de mama, posterior al diagnóstico en un centro de referencia oncológico de la ciudad de Medellín, Colombia

Métodos: estudio de cohorte retrospectiva realizado con los registros de pacientes con cáncer de mama $(n=141)$ atendidas un centro oncológico de referencia. Se midieron variables sociodemográficas, laborales, relacionadas con el tratamiento y de retorno al trabajo.

Resultados: la edad promedio al diagnóstico fue de $45.8 \pm 9$ años, La mayoría de las mujeres estaban en la premenopausia, el $45 \%$ realizaban trabajo manual. Los indicadores de mayor severidad de la enfermedad, así como el trabajo manual, la presencia de linfedema y un mayor número y días de incapacidad se relacionaron negativamente con el retorno al trabajo de estas pacientes. El 93\% de las pacientes retornaron al trabajo.

Conclusiones: el retorno al trabajo después de un cáncer de mama difiere según la severidad de la enfermedad, factores relacionados con el tratamiento y tipo de trabajo.

Palabras claves: Neoplasia de la mama; Reinserción al trabajo; Empleo; Ausencia por enfermedad; Complicaciones

\begin{abstract}
Introduction: Advances in the diagnosis and treatment of breast cancer have improved the prognosis for these patients. Consequently, a greater number of survivors are facing the process of returning to work. The objective of the present study was to analyze the frequency, median time and factors related to the return to work of patients with breast cancer, after diagnosis and completion of treatment at a cancer referral center in the city of Medellín, Colombia
\end{abstract}

Methods: a retrospective cohort study was carried out with registries of patients with breast cancer $(n=141)$ from a reference cancer center. Sociodemographic, occupational, treatment variables and prevalence of return to work were measured.

Results: The average age at diagnosis was $45.8 \pm 9$ years, Most of the women were premenopausal, and $45 \%$ performed manual labour. Advanced disease stage, manual labour, the presence of lymphedema and a greater number of episodes and days of disability were negatively related to return to work. A total of $93 \%$ of patients returned to work.

Conclusions: This study shows that return to work differs according to disease stage, treatment-related factors and type of work.

Keywords: return to work; breast cancer; sick leave; employment; complications 


\section{Introducción}

El cáncer de mama es la neoplasia que afecta con mayor frecuencia a las mujeres en general y específicamente a aquellas que se encuentran en edades laboralmente activas ${ }^{(1)}$. Para el caso de Colombia, la incidencia de cáncer de mama en las mujeres entre los 20 a 54 años es de 46.5 por cada 100.000(2). En España según las proyecciones de la Sociedad Española de Oncología Médica, se estima que el cáncer de mama será uno de los más frecuentemente diagnosticados para el año 2020, tendencia similar a la observada en 2019, con 32953 nuevos casos. ${ }^{(3)}$

Con los mejores métodos de tamizaje, diagnóstico y tratamientos más dirigidos de esta neoplasia, la supervivencia de estas pacientes ha aumentado( ${ }^{(4,5)}$. Este aumento en la supervivencia junto con el envejecimiento de la población activa( ${ }^{(6,7)}$, plantean el hecho de que cada vez más se incrementará el número de mujeres con cáncer de mama, que se enfrenten con el proceso de retorno al trabajo y como consecuencia del aumento en la esperanza de vida, estas pacientes tendrán que realizar su trabajo hasta una edad superior de retiro.

El retorno al trabajo es un proceso complejo que involucra las esferas física, psicológica y laboral de las pacientes y que se relaciona en gran medida con el sentido de regreso a la normalidad después de un proceso patológico, la seguridad de los ingresos y la participación social(8).

La evidencia científica al respecto ha relacionado algunos factores sociodemográficos; como la edad y el nivel educativo ${ }^{(0,10)}$, características laborales ${ }^{(11)}$, de la severidad de la enfermedad ${ }^{(12)}$ y del tipo de tratamiento y sus efectos adversos ${ }^{(13)}$ con el retorno al trabajo. No se dispone de artículos científicos que evalúen el retorno al trabajo en pacientes con cáncer de mama en el contexto colombiano.

El objetivo de este estudio es analizar la frecuencia, la mediana del tiempo y los factores relacionados con el retorno al trabajo de pacientes con cáncer de mama, posterior al diagnóstico en un centro de referencia oncológico de la ciudad de Medellín, Colombia.

\section{Métodos}

\section{Diseño y población de estudio}

Estudio de cohorte retrospectiva, en el que se analizó la información proveniente de historia clínica, de pacientes mayores de 18 años, con diagnóstico de enfermedad maligna de la mama, quienes reportaron estar activas laboralmente al momento del diagnóstico y que hubieran terminado en su totalidad el tratamiento para el cáncer primario en el año inmediatamente anterior al comienzo del estudio (2018), atendidas en una clínica oncológica $(n=141)$. Se trata de una entidad de alta complejidad con un modelo de atención integral para el paciente oncológico, teniendo como centro el paciente y su familia. Se excluyeron los registros de pacientes que no tuvieran la información sobre las características laborales $(n=3)$. 
Se recolectaron datos sobre las características sociodemográficas, laborales, clínicas, de tratamiento, tiempo del retorno al trabajo y las molestias reportadas por las mujeres que regresaron a realizar sus mismas tareas.

\section{Procedimiento}

Se recolectó la información clínica, demográfica y laboral de las pacientes mediante a partir del registro sistematizado de atenciones médicas. No se registró en ningún momento el nombre o documento de identificación para salvaguardar el anonimato de las mujeres participantes. El estudio y todos sus procedimientos fueron aprobados por el Comité de ética en Investigación de la institución dónde se realizó el estudio, código acta 61.

\section{Variables}

Variable dependiente: retorno al trabajo, definido como la paciente que habiendo culminado su tratamiento se encontrara activa laboralmente al momento del estudio.

Variables independientes: Características sociodemográficas: nivel educativo, clasificado en: primaria, secundaria, técnico/tecnológico (grado medio), universitario, posgrado. Estado civil recategorizado como: con pareja, sin pareja. Edad al diagnóstico en años cumplidos. Paridad medida como el número total de embarazos que ha tenido la mujer, incluyendo los abortos ${ }^{(14)}$. Características laborales: ocupación se agrupó según el autoreporte de la paciente en: administrativo, comercio y ventas, salud y laboratorios, operaria, limpieza y oficios varios, docencia, estilista y otros (en donde se agrupan categorías de menor frecuencia). El trabajo manual se definió según lo reportado por el Grupo de trabajo de la Sociedad Española de Epidemiología y de la Sociedad Española de Medicina de Familia y Comunitaria, en su propuesta de medida de la clase social, clasificaciones IVa, IVb y $\bigvee^{(15)}$ esto se realizó mediante la correlación de la actividad económica de la empresa y la ocupación reportada por la paciente y que figuraba en la historia .Características clínicas: el estado menopaúsico se categorizó de forma dicotómica (premenopausia o postmenopausia). El tipo histológico se registró de acuerdo con lo propuesto por Burstein et al(16), en ductal, lobulillar y las categorías con baja frecuencia se agruparon en otros. Se utilizó la clasificación del subtipo molecular propuesta por Perou et al ${ }^{(17)}$ en Luminal A, Luminal B, Her-2 enriquecido y Triple negativo. El grado histológico se registró tal como lo propone el National Cancer Institute ${ }^{(18)}$ en alto, moderado o bajo (el estado menopaúsico, el tipo histológico, subtipo molecular y grado histológico.

Características del tratamiento: tipo de tratamiento recibido: se clasificó según lo indicado en la historia clínica en: cirugía, quimioterapia, radioterapia, terapia endocrina y las combinaciones de tratamientos más frecuentes. Los efectos adversos del tratamiento se registraron según las fichas de seguimiento oncológico de las pacientes molestias, para esto se contabilizo el total de mujeres que refirieron alguna molestia del tratamiento sobre el total de mujeres que recibieron dicho trata- 
miento. Para cada uno de los casos se presenta la molestia reportada con mayor frecuencia.

\section{Análisis de los datos}

Se diseñó una plantilla en Microsoft Excel en donde se depositó la información de las variables independientes y la variable de retorno al trabajo. Se utilizaron estadísticos descriptivos para conocer la distribución de las variables sociodemográficas, clínicas, y laborales. El tiempo total de retorno al trabajo se calculó como la diferencia entre la fecha de retorno al trabajo menos la fecha de la incapacidad posterior al tratamiento, más 365 días. La mediana del tiempo al retorno al trabajo se calculó a través del método de Kaplan Meier y las comparaciones entre grupos mediante la prueba del Log Rank test. La proporción de mujeres que retornaron al trabajo a los 3, 6 y 12 meses se calculó utilizando tablas de mortalidad; en el caso de las censuras se tomó en cuenta el dato del tiempo incompleto aportado por dicho participante antes de ser censurado y se excluyeron para el siguiente momento.

Todos los análisis estadísticos se realizaron en SPSS versión 21. Todas las pruebas estadísticas se calcularon con una significación del 5\%.

\section{Resultados}

El análisis de los datos permitió identificar que 141 pacientes se encontraban activas laboralmente al momento del diagnóstico y que terminaron su tratamiento en el año inmediatamente anterior al comienzo del estudio, con un promedio de edad de 45,8 \pm 9,0 al momento del diagnóstico. La mayoría de las mujeres tenían un nivel educativo medio (técnica/tecnología) (36.1\%). El 53.9\% de ellas refirieron no tener pareja. La paridad promedio fue de $1.26 \pm 1.0$. Con respecto a las características laborales, el $45.4 \%$ realizan un trabajo manual y las ocupaciones con mayor frecuencia son las del área administrativa. Esta información aparece en la Tabla 1.

Con respecto a las características clínicas de las pacientes, se puede observar que el $87.2 \%$ se encuentran en la premenopausia, con una predominancia del tipo histológico ductal y el subtipo molecular Luminal B, con un grado histológico bajo (35.5\%) -Tabla 2-.

En la Tabla 3 se presenta la frecuencia de cada uno de los tratamientos recibidos por las pacientes en estudio, la frecuencia de las complicaciones de cada tratamiento y el tipo de complicación más frecuente, en donde se observa que la mayoría de las pacientes son sometidas a cirugía (94.3\%), presentando complicaciones el 38.3\% de ellas, siendo lo más frecuente la disfunción de hombro. En las combinaciones de tratamiento más frecuentemente indicadas a las pacientes se observa una mayor frecuencia de complicaciones, en su mayoría la disfunción de hombro. En total, 127 pacientes (93\%) retornaron a su trabajo luego de haber finalizado su tratamiento, nueve pacientes perdieron su trabajo y en cinco casos no fue posible determinar el desenlace laboral. 
Tabla 1. Características sociodemográficas de las mujeres participantes del estudio

\begin{tabular}{|c|c|}
\hline Característica & $\mathrm{n}(\%)$ \\
\hline \multicolumn{2}{|l|}{ Nivel educativo } \\
\hline Primaria & $12(8,5)$ \\
\hline Secundaria & $41(29,1)$ \\
\hline Técnico/Tecnológico (grado medio) & $51(36,1)$ \\
\hline Universitaria & $27(19,1)$ \\
\hline Posgrado & $10(7,1)$ \\
\hline \multicolumn{2}{|l|}{ Estado civil } \\
\hline Con pareja & $65(46,1)$ \\
\hline Sin pareja & $76(53,9)$ \\
\hline Edad al diagnóstico (promedio \pm DS) & $45,8 \pm 9,0$ \\
\hline Paridad (promedio \pm DS) & $1,26 \pm 1,0$ \\
\hline Trabajo manual & $64(45,4)$ \\
\hline \multicolumn{2}{|l|}{ Ocupación } \\
\hline Administración & $52(36,9)$ \\
\hline Comercio y ventas & $22(15,6)$ \\
\hline Salud y laboratorio clínico & $10(7,1)$ \\
\hline Operaria & $14(9,9)$ \\
\hline Limpieza y oficios varios & $13(9,2)$ \\
\hline Docencia & $7(5)$ \\
\hline Estilista & $6(4,3)$ \\
\hline Otras & $17(12)$ \\
\hline
\end{tabular}

Tabla 2. Características clínicas de las mujeres participantes del estudio

\begin{tabular}{ll}
\hline Característica & $\mathrm{n}(\%)$ \\
\hline
\end{tabular}

\section{Estado menopáusico}

Premenopaúsica

\section{Tipo histológico}

Ductal

Lobulillar

Otro

\section{Subtipo molecular}

Luminal A

Luminal B

Her 2 enriquecido

Triple negativo

\section{Grado histológico}

\begin{tabular}{lr} 
I (bajo) & $50(35,5)$ \\
II (moderado) & $47(33,3)$ \\
III (alto) & $42(29,8)$ \\
\hline
\end{tabular}


Tabla 3. Tratamientos recibidos por las mujeres participantes, frecuencia y tipo de las complicaciones posteriores al tratamiento, en un centro de referencia en la ciudad de Medellín

\begin{tabular}{|c|c|c|c|}
\hline Tratamiento & Frecuencia (\%) & Complicaciones (\%) ${ }^{a}$ & $\begin{array}{l}\text { Complicación más } \\
\text { frecuente }(\%)^{b}\end{array}$ \\
\hline Cirugía & $94.30 \%$ & $38.30 \%$ & $\begin{array}{l}\text { Disfunción de hombro } \\
15.0 \%\end{array}$ \\
\hline Quimioterapia & $70.20 \%$ & $31 \%$ & Neuropatía 12,12\% \\
\hline Radioterapia & $74.05 \%$ & $9.52 \%$ & Radiodermatitis 4,76\% \\
\hline Terapia endocrina & $61.70 \%$ & $13.76 \%$ & $\begin{array}{l}\text { Hemorragia uterina } \\
\text { anormal 2,1\% }\end{array}$ \\
\hline Cirugía + Quimioterapia & $66.66 \%$ & $53.19 \%$ & $\begin{array}{l}\text { Disfunción de hombro } \\
16.0 \%\end{array}$ \\
\hline Cirugía + Radioterapia & $72.34 \%$ & $50.0 \%$ & $\begin{array}{l}\text { Disfunción de hombro } \\
36.0 \%\end{array}$ \\
\hline $\begin{array}{l}\text { Cirugía + Terapia } \\
\text { hormonal }\end{array}$ & $60.28 \%$ & $57 \%$ & Disfunción de hombro 36\% \\
\hline
\end{tabular}

Cuando se observan los factores que se relacionan con las medianas de retorno al trabajo de estas pacientes, es importante resaltar que realizar trabajo manual, un estadio tumoral avanzado, haber recibido quimioterapia y presentar linfedema, presentan diferencias estadísticamente significativas (Tabla 4).

Para la totalidad de las pacientes que se reincorporaron a su trabajo, la mediana de tiempo fue de $12 \mathrm{RIQ}=7.4-16.5$ semanas. La curva de Kaplan Meier muestra que las medianas de tiempo al retorno al trabajo son diferentes de acuerdo con el hecho de que el trabajo realizado por la paciente tenga trabajo manual, siendo para estas pacientes de 16 (7.3-24.66) semanas, mientras que para las pacientes cuyo trabajo no es manual el tiempo de retorno es menor; 8 (3.91-12.08) (Figura 1).

Al analizar a las mujeres que regresaron al trabajo acerca de las molestias sentidas al momento de realizar su trabajo, cerca del $54 \%$ de ellas refieren tener alguna, se evidencia también que aproximadamente 1 de cada 5 pacientes refieren dolor en miembro superior o espalda. En menor proporción se reporta la disminución o pérdida de la movilidad en miembro superior (Figura 2). 
Tabla 4. Proporción de retorno al trabajo (RTT) a los 3, 6 y 12 meses posteriores al tratamiento y medianas de tiempo de retorno al trabajo según diversos factores

\begin{tabular}{|c|c|c|c|c|c|}
\hline \multirow[t]{2}{*}{ Característica } & \multirow{2}{*}{$\begin{array}{l}\text { RTT a los } 3 \\
\text { meses }\end{array}$} & \multirow{2}{*}{$\begin{array}{l}\text { RTT a los } 6 \\
\text { meses } \\
\text { \%RTT }\end{array}$} & \multirow{2}{*}{$\begin{array}{l}\text { RTT } 12 \\
\text { meses } \\
\text { \%RTT }\end{array}$} & \multirow{2}{*}{$\begin{array}{l}\text { Mediana del } \\
\text { tiempo al retorno } \\
(\text { IC95\%) }\end{array}$} & \multirow[t]{2}{*}{$\mathbf{p}^{\mathrm{b}}$} \\
\hline & & & & & \\
\hline \multicolumn{6}{|l|}{ Tipo de trabajo } \\
\hline Manual & $32 / 97$ & $9 / 28$ & 19/19 & $21,24(18,8-32,42)$ & 0,035 \\
\hline No manual & $52 / 107$ & $9 / 20$ & $8 / 12$ & $10,82(3,09-12,08)$ & \\
\hline \multicolumn{6}{|l|}{ Cirugía } \\
\hline $\mathrm{Si}$ & $82 / 133$ & $15 / 46$ & $27 / 30$ & $12(7,30-16,70)$ & 0,406 \\
\hline No & $2 / 8$ & $1 / 2$ & $0 / 1$ & $32(0,80-63,19)$ & \\
\hline \multicolumn{6}{|l|}{ Quimioterapia } \\
\hline Si & $47 / 99$ & $15 / 46$ & $26 / 30$ & $22(19,04-24,95)$ & 0,000 \\
\hline No & $37 / 42$ & $1 / 2$ & $1 / 1$ & $4(3,35-4,65)$ & \\
\hline \multicolumn{6}{|l|}{ Radioterapia } \\
\hline $\mathrm{Si}$ & $62 / 105$ & $13 / 38$ & $23 / 25$ & $12(5,46-18,53)$ & 0,605 \\
\hline No & $22 / 36$ & $3 / 10$ & $4 / 6$ & $10(6,30-13,69)$ & \\
\hline \multicolumn{6}{|c|}{ Terapia endocrina } \\
\hline $\mathrm{Si}$ & $48 / 87$ & $10 / 25$ & $13 / 15$ & $8(4,49-11,50)$ & 0,073 \\
\hline No & $17 / 54$ & $6 / 23$ & $14 / 16$ & $20(14,95-25,04)$ & \\
\hline \multicolumn{6}{|c|}{ Estadio Tumoral } \\
\hline 0 & $5 / 7$ & $2 / 2$ & 0 & $4,00(1,46-6,53)$ & 0,002 \\
\hline । & $36 / 43$ & $2 / 6$ & $4 / 4$ & $6,00(2,96-9,04)$ & \\
\hline ॥ & $23 / 46$ & $8 / 22$ & $11 / 13$ & $20,00(10,07-29,92)$ & \\
\hline III & $14 / 29$ & $5 / 15$ & $9 / 10$ & $24,00(7,19-40,80)$ & \\
\hline IV & $2 / 7$ & $1 / 4$ & $2 / 3$ & $24,00(7,19-40,80)$ & \\
\hline \multicolumn{6}{|l|}{ Linfedema } \\
\hline $\mathrm{Si}$ & $3 / 17$ & $5 / 11$ & $5 / 6$ & $24(20,11-27,88)$ & 0,023 \\
\hline No & $62 / 116$ & $10 / 35$ & $21 / 24$ & $8(4,88-11,11)$ & \\
\hline \multicolumn{6}{|c|}{ RTT: retorno al trabajo } \\
\hline \multicolumn{6}{|c|}{ Mediana en semanas } \\
\hline log rank test & & & & & \\
\hline
\end{tabular}




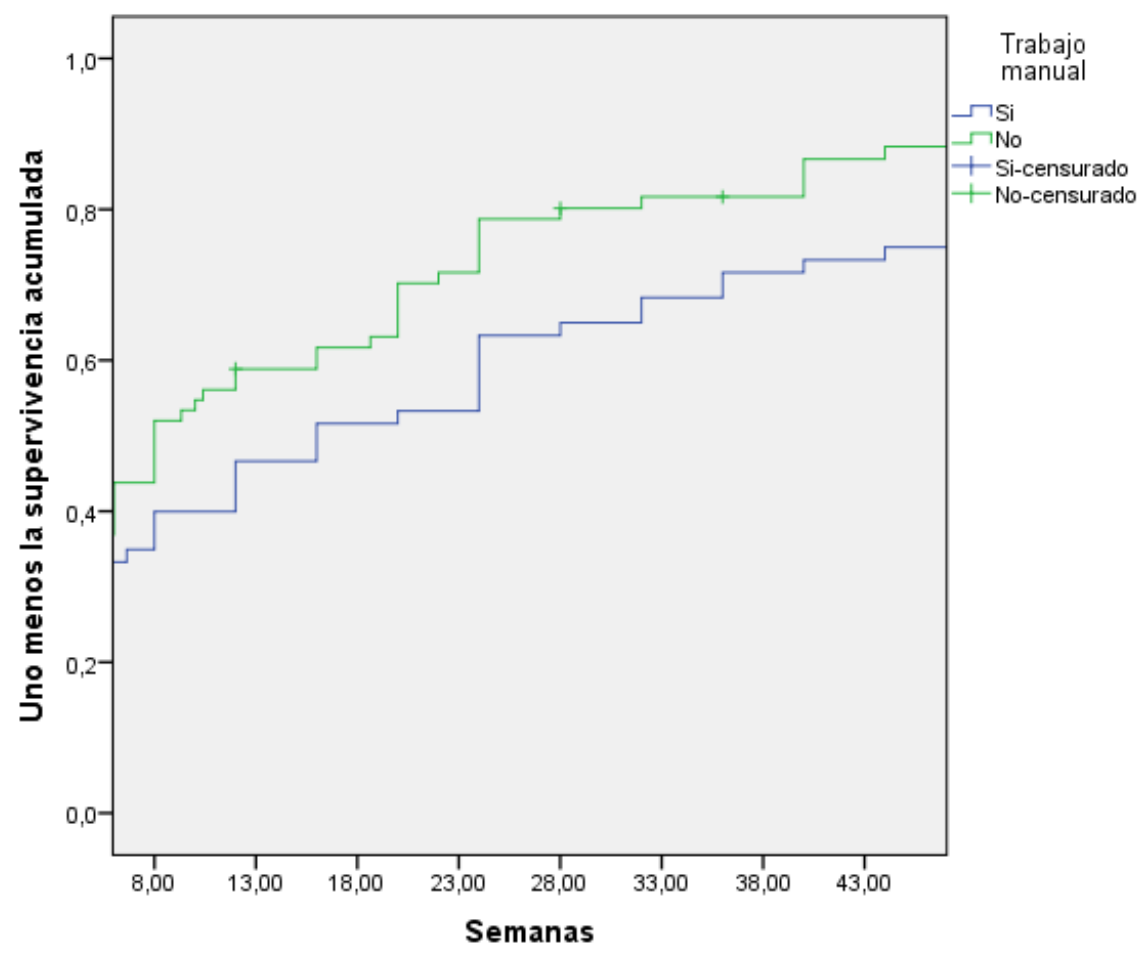

Figura 1. Curva de Kaplan Meier para la mediana del tiempo de retorno al trabajo posterior al tratamiento, según trabajo manual

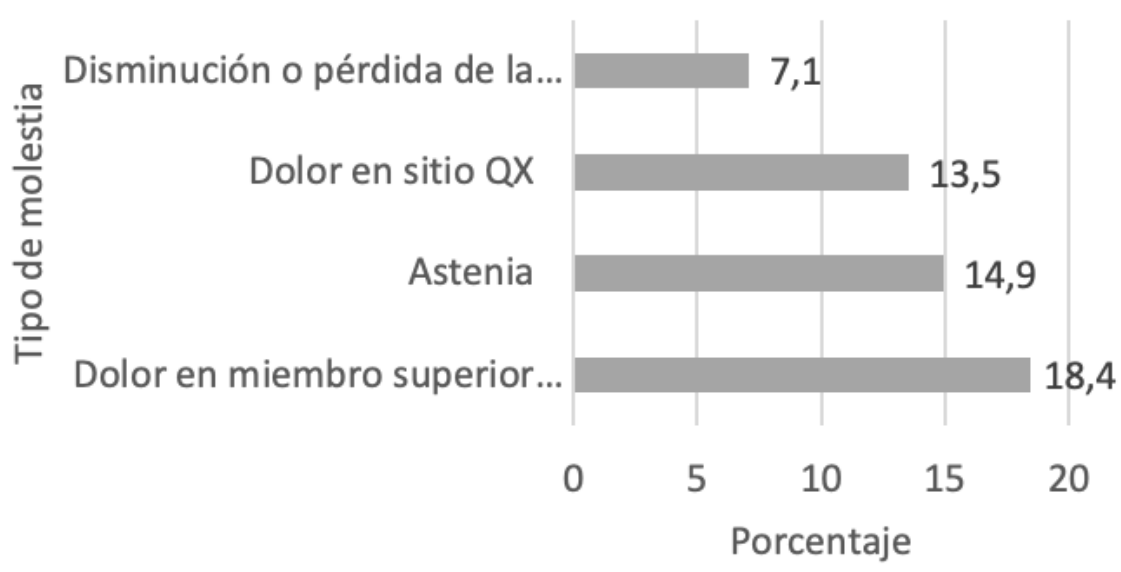

Figura 2. Porcentaje de aparición de las molestias sentidas al realizar el trabajo reportadas por las mujeres que retornaron al trabajo 


\section{Discusión}

El hallazgo principal de este estudio es el dato de una frecuencia del retorno al trabajo del $93 \%$ en pacientes quienes habían culminado su tratamiento para cáncer de mama en el año inmediatamente anterior al comienzo del presente estudio. Los factores asociados al retorno al trabajo en este estudio fueron los indicadores de severidad de la enfermedad, haber recibido cirugía, tener linfedema y un trabajo manual. No se asoció el hecho de recibir quimio o radioterapia, la edad, ni el estado civil de la trabajadora.

La frecuencia encontrada de retorno al trabajo fue del 93\%, una cifra superior a la de otros estudios realizados en pacientes con cáncer de mama ${ }^{(19,20)}$, pero similar a los resultados de Goss et al en trabajadoras del sector salud(21). Las explicaciones potenciales a esta cifra, pueden ser diversas; incluyendo un promedio inferior de edad, lo que impide el retiro temprano, mayor frecuencia de empleo informal y autoempleo, que a pesar de que en la fuente de información no se encuentra específicamente este dato desagregado, se infiere tal de lo que se conoce del contexto colombiano, en donde para el trimestre de julio a septiembre de 2019, la proporción de informalidad laboral en las mujeres se ubicó en el $48.3 \%{ }^{(22)}$. Una jornada flexible de trabajo también se ha relacionado con una mayor prevalencia de retorno al trabajo(23), esta situación ocurre en el los trabajos informales y de autoempleo.

Un análisis de las características sociodemográficas de este grupo de pacientes reflejan una población de edad media, con un promedio de $45 \pm 9$ años, bastante similar a lo encontrado para mujeres iraníes reclutadas de cuatro centros de referencia oncológica, en quienes el promedio de edad fue de $44.3 \pm 6.72$ años $^{(10)}$, pero inferior a la edad de pacientes incluidas en series realizadas en Europa y Estados Unidos, en los que se observan promedios superiores a los 52 años ${ }^{(24-26)}$. Con respecto a los factores asociados con este desenlace, la edad no presentó diferencias estadísticamente significativas entre las mujeres que retornaron a su trabajo y las que no lo hicieron, resultado consistente con lo que se reporta en el meta-análisis de estudios observacionales realizado por Wang et al ${ }^{(27)}$. Sin embargo, al realizar esta comparación es importante anotar que otros reportes establecen que algunas mujeres que se encuentran muy cerca de su edad de retiro, prefieren realizar este de forma anticipada ${ }^{(28)}$, lo que tiene una implicación directa en la prevalencia de la reincorporación laboral. Sin embargo, es importante anotar que existen diferencias contextuales no evaluadas, tales como el tipo de sistema de salud, que pueden explicar el efecto de la edad sobre el retorno al trabajo.

El meta-análisis de Wang et al(27), además, no encuentra diferencias según el estado civil, en concordancia a lo encontrado en el presente estudio. El nivel educativo tampoco presentó diferencias significativas entre las mujeres que retornaron y las que no lo hicieron, resultados consistentes con lo reportado por Bouknight et al(11), pero en contraposición con lo manifestado en otros estudios ${ }^{(29,30)}$.

Los indicadores de severidad de la enfermedad se relacionaron de forma negativa con la probabilidad de retorno al trabajo, a mayor tamaño tumoral, mayor número 
de ganglios comprometidos y la presencia de metástasis disminuyen la frecuencia de este retorno, consistente con los estudios incluidos en la revisión sistemática de Islam et al ${ }^{(31)}$.

El tratamiento con quimioterapia y/o radioterapia no se relacionó con una menor probabilidad de retorno al trabajo, en concordancia con Lee et al ${ }^{(32)}$, cuando evalúan este desenlace a los tres años post tratamiento. No obstante, los hallazgos en torno a la quimioterapia son contradictorios entre estudios. El hecho de recibir cirugía se relacionó negativamente con el retorno al trabajo, en correspondencia con lo descrito por Muhajid et al en una muestra de mujeres de Los Ángeles, pero sobrerepresentada de latinas y afroamericanas ${ }^{(33)}$. El lifedema, una complicación frecuente de estas pacientes, se asocia con una menor probabilidad de retorno al trabajo, conocido ya por tener efectos negativos adicionales a los de la enfermedad y el tratamiento sobre la empleabilidad y la capacidad funcional para realizar el trabajo ${ }^{(34)}$.

Entre las pacientes que retornaron a su trabajo, cerca del 54\% manifiesta tener molestias al realizar su trabajo, la mayor parte de ellas en miembro superior o espalda, en proporciones similares a lo encontrado por Assis et al(35). Estas molestias pueden en un momento posterior del tiempo influir en el retiro del trabajo de estas pacientes.

Cuando el trabajo de la paciente es manual se deben analizar las mejores opciones de tratamiento para un pronto retorno al trabajo, en este punto es importante anotar que tanto la legislación española en el artículo 25 de su Ley de Prevención de Riesgos Laborales y la legislación colombiana en la Ley 776 de 2002, establecen con claridad cuáles son las acciones y obligaciones de un empleador cuando se enfrenta a la reubicación de un trabajador con restricciones para ejecutar sus tareas $^{(36,37)}$

Según el conocimiento de los autores, este es el primer estudio que analiza la reincorporación laboral de pacientes en Colombia, en un contexto diferente a los europeos o anglosajones, donde la informalidad laboral y el trabajo manual son menores que un país en vía de desarrollo, lo que para nosotros representa una fortaleza del estudio.

No obstante, los resultados deben ser analizado a la luz de las siguientes limitaciones; en primer lugar los resultados no son representativos a nivel poblacional dado que la información de las pacientes proviene de un único centro de referencia oncológico, también es importante tener en cuenta que en el momento del diagnóstico, estas pacientes se encontraban trabajando, por tanto son de menor edad y mejor estado de salud que la población general. No se tiene el dato desagregado de cuantas de las mujeres que retornan al trabajo lo hacen en el sector informal de la economía o como autoempleadas. La comparación de las cifras de retorno al trabajo puede estar marcada por las diferencias contextuales del sistema de salud y con las prestaciones económicas que se derivan de las incapacidades médicas, que a menudo no son tenidas en cuenta en los diferentes estudios, lo que impide un análisis al respecto. 
Los resultados del presente estudio muestran que la cirugía para cáncer de mama es uno de los factores que se relaciona negativamente con el retorno al trabajo, esto en parte, debido a las complicaciones presentadas por las pacientes, que limitan su capacidad laboral. Es por esto que se sugiere a los clínicos reducir en lo posible los tratamientos agresivos y discutir con la paciente el impacto que cada opción de tratamiento tiene sobre los desenlaces laborales, para lograr decisiones informadas.

Con respecto a los tomadores de decisiones, es claro que la normatividad laboral de los países establece acciones y obligaciones por parte del empleador para la protección de los trabajadores sensibles, permitiendo flexibilidad laboral, reubicación y readaptación, estrategias que deben ser vigiladas en los lugares de trabajo.

Se requieren de nuevas investigaciones con una temporalidad mayor a la de este estudio permitirán observar el impacto de los factores relacionados con el retorno al trabajo. De interés especial para futuras investigaciones son las pacientes jóvenes, puesto que a pesar de que ellas tienen el mayor potencial de años laborales, a menudo, reciben las opciones de tratamiento más agresivas. A futuro también se requiere conocer cuáles son las adaptaciones de puestos de trabajos para las pacientes luego del cáncer de mama, con limitaciones en la capacidad laboral, que se reincorporan al trabajo y cuál es el apoyo social en el trabajo que reciben.

Como conclusión se puede decir que la prevalencia de retorno al trabajo en este estudio fue superior a la de otros reportes. Los indicadores de severidad de la enfermedad se relacionaron negativamente con el retorno al trabajo. Recibir quimioterapia o radioterapia no se asoció con el retorno al trabajo.

\section{Bibliografía}

1. American Cancer Society. Cancer facts and statistics 2018 [cited 2018 05/07/2018]. Available from: https://www.cancer.org/content/dam/cancer-org/ research/cancer-facts-and-statistics/annual-cancer-facts-and-figures/2018/cancer-facts-and-figures-special-section-ovarian-cancer-2018.pdf.

2. International Agency for Research on Cancer. Estimated age-standardized incidence rates (World) in 2018, Colombia, females, all ages Lyon2019 [cited 2019 30/10/2019]. Available from: https://gco.iarc.fr/today/online-analysis-multi-bar$\mathrm{s} ? \mathrm{v}=2018$.

3. Sociedad Española de Oncología Médica. Las cifras del cáncer en España 2020 España: Sociedad Española de Oncología Médica; 2019 [citado 2020 13/04]. Disponible en: https://seom.org/seomcms/images/stories/recursos/Cifras_del_cancer_2020.pdf.

4. de Boer AG, Taskila T, Ojajarvi A, van Dijk FJ, Verbeek JH. Cancer survivors and unemployment: a meta-analysis and meta-regression. JAMA. 2009;301(7):753-62.

5. Gilbert SM, Miller DC, Hollenbeck BK, Montie JE, Wei JT. Cancer survivorship: challenges and changing paradigms. J Urol. 2008;179(2):431-8. 
6. Atella V, Piano Mortari A, Kopinska J, Belotti F, Lapi F, Cricelli C, et al. Trends in age-related disease burden and healthcare utilization. Aging Cell. 2019;18(1):e12861-e.

7. Fontana L, Kennedy BK, Longo VD, Seals D, Melov S. Medical research: treat ageing. Nature. 2014;511(7510):405-7.

8. Heuser C, Halbach S, Kowalski C, Enders A, Pfaff H, Ernstmann N. Sociodemographic and disease-related determinants of return to work among women with breast cancer: A German longitudinal cohort study. BMC Health Services Research. 2018;18:1000-10.

9. Peugniez C, Fantoni S, Leroyer A, Skrzypczak J, Duprey M, Bonneterre J. Return to work after treatment for breast cancer: single center experience in a cohort of 273 patients. Bulletin du Cancer. 2011;98(7):E69-E79.

10. Azarkish F, Mirzaii Najmabadi K, Latifnejad Roudsari R, Homaei Shandiz F. Factors Related to Return to Work in Women After Breast Cancer in Iran. Iran Red Crescent Med J. 2015;17(9):e19978.

11. Bouknight RR, Bradley CJ, Luo Z. Correlates of Return to Work for Breast Cancer Survivors. Int J Clin Oncol. 2006;24(3):345-53.

12. Cheung K, Ching SYS, Chan A, Cheung D, Cheung SYP. The impact of personal-, disease- and work-related factors on work ability of women with breast cancer living in the community: a cross-sectional survey study. Support Care Cancer. 2017;25(11):3495-504.

13. Drolet M, Maunsell E, Mondor M, Brisson C, Brisson J, Mâsse B, et al. Work absence after breast cancer diagnosis: a population-based study. Can Med Assoc J. 2005;173(7):765-71.

14. Instituto Nacional de Gestión Sanitaria. Manual básico de Obstetricia y Ginecología. Subdirección General de Gestión Económica y Recursos Humanos, editor. Madrid: Instituto Nacional de Gestión Sanitaria; 2017. 388 p.

15. Grupo de trabajo de la Sociedad Española de Epidemiología y de la Sociedad Española de Medicina de Familia y Comunitaria. Una propuesta de medida de la clase social. Atención Primaria. 2000;25(5):350-63.

16. Burstein HJ HJ, Morrow M,. Malignant Tumors on the breast. Cancer: Principles \& Practice of Oncology. 8. Philadelphia: de Vita, Hellman, Rosemberg; 2008. p. $1606-54$.

17. Perou CM, Sørlie T, Eisen MB, van de Rijn M, Jeffrey SS, Rees CA, et al. Molecular portraits of human breast tumours. Nature. 2000;406(6797):747-52.

18. National Cancer Institute. Diagnóstico y estadificacicón Bethesda2013 [citado 2020 02/03]. Disponible en: https://www.cancer.gov/espanol/cancer/diagnostico-estadificacion/estadificacion. 
19. Ahn E, Cho J, Shin D, Park B, Ahn S-H, Noh D-Y, et al. Impact of breast cancer diagnosis and treatment on work-related life and factors affecting them. Breast Cancer Res Treat. 2009;116:609-16.

20. Noeres D, Park-Simon T-W, Grabow J, Sperlich S, Koch-Gießelmann H, Jaunzeme J, et al. Return to work after treatment for primary breast cancer over a 6-year period: results from a prospective study comparing patients with the general population. Support Care Cancer. 2013;21(7):1901-9.

21. Goss $C$, Leverment IM, de Bono AM. Breast cancer and work outcomes in health care workers. Occupational medicine (Oxford, England). 2014;64(8):635-7.

22. Departamento Administrativo Nacional de Estadística. Medición de empleo informal y seguridad social Santafe de Bogotá2019 [citado 2020 13/04]. 1-14]. Disponible en: https://www.dane.gov.co/files/investigaciones/boletines/ech/ech_informalidad/bol_ech_informalidad_jul19_sep19.pdf.

23. Jagsi R, Abrahamse PH, Lee KL, Wallner LP, Janz NK, Hamilton AS, et al. Treatment decisions and employment of breast cancer patients: Results of a population-based survey. Cancer. 2017;123(24):4791-9.

24. Hedayati E, Johnsson A, Alinaghizadeh H, Schedin A, Nyman H, Albertsson M. Cognitive, psychosocial, somatic and treatment factors predicting return to work after breast cancer treatment. Scand J Caring Sci. 2013;27(2):380-7.

25. Ross L, Petersen MA, Johnsen AT, Lundstroem LH, Carlsen K, Groenvold M. Factors associated with Danish cancer patients' return to work. A report from the population-based study 'The Cancer Patient's World'. Cancer Epidemiology. 2012;36(2):222-9.

26. Tison A, Sagaon-Teyssier L, Sansonetti C, Blatier J-F, Paraponaris A, Aparicio T, et al. Transitions in the labor market after cancer: a comparison of self-employed workers and salaried staff. Supportive Care in Cancer. 2016;24(12):4879-86.

27. Wang L, Hong BY, Kennedy SA, Chang Y, Hong CJ, Craigie S, et al. Predictors of Unemployment After Breast Cancer Surgery: A Systematic Review and Meta-Analysis of Observational Studies. J Clin Oncol. 2018;36(18):1868-79.

28. Lindbohm ML, Kuosma E, Taskila T, Hietanen P, Carlsen K, Gudbergsson S, et al. Early retirement and non-employment after breast cancer. Psycho-Oncology. 2014;23(6):634-41.

29. Petersson L-M, Wennman-Larsen A, Nilsson M, Olsson M, Alexanderson $K$. Work situation and sickness absence in the initial period after breast cancer surgery. Acta Oncologica. 2011;50(2):282-8.

30. Fantoni SQ, Peugniez C, Duhamel A, Skrzypczak J, Frimat P, Leroyer A. Factors related to return to work by women with breast cancer in northern France. J Occup Rehabil. 2010;20(1):49-58. 
31. Islam T, Dahlui M, Majid HA, Nahar AM, Mohd Taib NA, Su TT. Factors associated with return to work of breast cancer survivors: a systematic review. BMC Public Health. 2014;14 Suppl 3:S8-S.

32. Lee MK, Kang HS, Lee KS, Lee ES. Three-Year Prospective Cohort Study of Factors Associated with Return to Work After Breast Cancer Diagnosis. Journal of occupational rehabilitation. 2017;27(4):547-58.

33. Mujahid MS, Janz NK, Hawley ST, Griggs JJ, Hamilton AS, Katz SJ. The impact of sociodemographic, treatment, and work support on missed work after breast cancer diagnosis. Breast Cancer Research and Treatment. 2010;119(1):213-20.

34. Boyages J, Kalfa S, Xu CY, Koelmeyer L, Mackie H, Viveros H, et al. Worse and worse off: the impact of lymphedema on work and career after breast cancer. SpringerPlus. 2016;5:657-65.

35. Assis MR, Marx AG, Magna LA, Ferrigno ISV. Late morbidity in upper limb function and quality of life in women after breast cancer surgery. Braz J Phys Ther. 2013;17:236-43.

36. Congreso de la República de Colombia. Ley 776 Organización, administración y prestaciones del Sistema General de Riesgos Profesionales. Santafé de Bogotá2002 [citado 2020 13/04]. Disponible en: https://www.funcionpublica.gov.co/ eva/gestornormativo/norma.php?i=16752.

37. Boletín Oficial del Estado. Ley 31/1995 de 8 de Noviembre, de Prevención de Riesgos Laborales. España1995 [citado 2020 13/04]. Disponible en: https://www. boe.es/buscar/act.php?id=BOE-A-1995-24292.

38. Greene FL PD, Fleming ID, et al. Breast. In: Springer, editor. AJCC Cancer Staging Manual. 6 ed. New York: Springer; 2002. p. 223-40. 